\title{
Axial-flux PM Wind Generator with A Soft Magnetic Composite Core
}

\author{
Yicheng Chen \\ Siemens \\ Atlanta \\ yicheng.chen@siemens.com
}

\author{
Pragasen Pillay, FIEEE \\ Department of Electrical \& Computer Engineering \\ Clarkson University \\ Potsdam, NY 13699-5720, USA \\ pillayp@clarkson.edu
}

\begin{abstract}
This paper presents the potential application of soft magnetic composite (SMC) material in low speed, directly driven, axial-flux permanent magnet (PM) wind generators. Comparative design studies are conducted on PM wind generators of different configurations with both lamination cores and SMC core. Finite element analysis is used to enhance the design precision, from which analytical formulas are modified. Through careful design an axial-flux PM wind generator with slotted SMC core is built and tested, demonstrating the advantages of better performance, reduced size and weight, low part count and low cost.
\end{abstract}

Keywords-axial-flux, outer rotor, permanent magnet, radialflux, soft magnetic composite, wind generator.

\section{INTRODUCTION}

In low speed, directly driven wind systems, PM axial-flux machines with slotted cores have proven to be superior to machines with other topologies in many aspects, such as high efficiency, short length and high torque /volume ratio [1]. The crucial issue to build this kind of machine is to form the armature core with slots and teeth. Solid steel cannot be used because of eddy currents. If common electrical steel is used the lamination must be wound in a spiral fashion to form a ring. It is difficult to cut the slots either before or after the ring being formed.

The group of soft magnetic composites (SMC) have been relatively recently expanded to include new electromagnetic materials with significantly improved low-medium frequency properties [2]. This has made SMC a viable alternative to steel laminations for a range of new applications, especially axial-flux electrical machines. The basis for soft magnetic composites is bonded iron powder. The powder particles are coated with an electrically insulated layer, which produces high electrical resistivity. The coated powder is then pressed into a solid material using a die and finally heat treated to anneal and cure the bond [3].

Compared with electrical steels widely used in electrical machines, the major advantage of the SMC is the material isotropic magnetic property in three dimensions, which opens up crucial magnetic circuit design benefits [4]. Another advantage of SMC is manufacturing. It is easy to make complex shapes while maintaining good dimensional tolerances, thus large volume manufacturing of low cost electromagnetic devices are possible. In addition, the powdered nature of the material yields low eddy current losses, which benefits machine efficiency. However, the permeability of this material is poor; saturation flux density is reduced as compared to electrical steel; high hysteresis loss can result from pressing strain and poor domain structures; the material strength depends on the epoxy bond, which makes the material weak; the nature of the binder makes the material brittle; porosity requires consideration in terms of corrosion protection.

In general, the properties of SMC show that directly replacing electrical steels with SMC is not reasonable. New design concept and structures should be sought to obtain at least comparable results.

This paper opens up the application of SMC to low speed, directly driven PM wind generators. It exploits the potential application of SMC in PM axial-flux constructions for which the magnetic reluctance of the magnets dominate the magnetic circuit, making such constructions less sensitive to the low permeability of the armature core and making best use of the flexibility of shaping and magnetic isotropy of this kind of material. Through carefully design, the advantages such as better performance, reduced size and weight, fewer parts and lower cost of axial-flux wind generators using SMC can be obtained. The details of the design and manufacture of a PM axial-flux wind generator with slotted SMC core will be discussed. The prototype was built at the Clarkson University machine shop. The prototype was tested and some experimental results are reported.

\section{MACHINE STRUCTURE}

Fig.1 shows the magnetic property and loss property comparison between the electrical steel and SMC material. It is easy seen that SMC material is inherently poorer than electrical steel about these properties. To compensate for these differences, the SMC machine must be designed using short magnetic path length and minimum weight.

Of all the topologies of PM wind generators discussed in the literature [1][5], the double rotor, axial-flux, and slotted structure is one of the best suited for the application of SMC, as shown in Fig. 2. In this structure, the flux starts from one pole mounted on the first disk, passing through air-gap and reaching the stator core with two teeth in series, separated by a yoke, then 


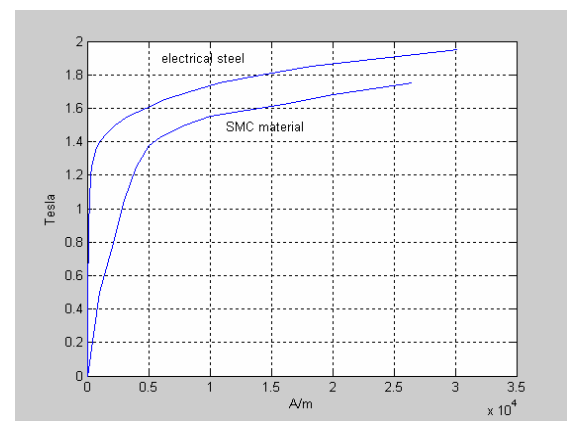

(a) B-H curve

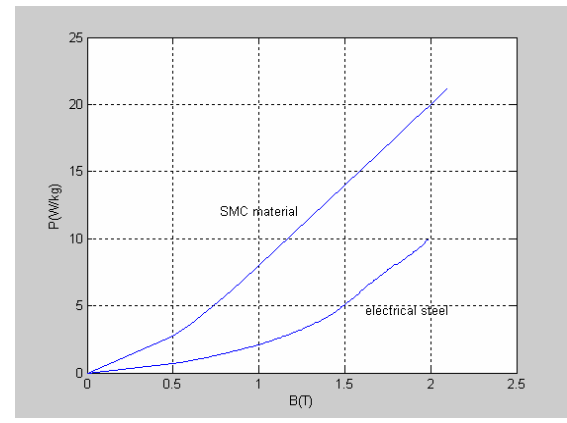

(b) Loss curve

Fig.1 Property comparison between electrical steel and SMC material

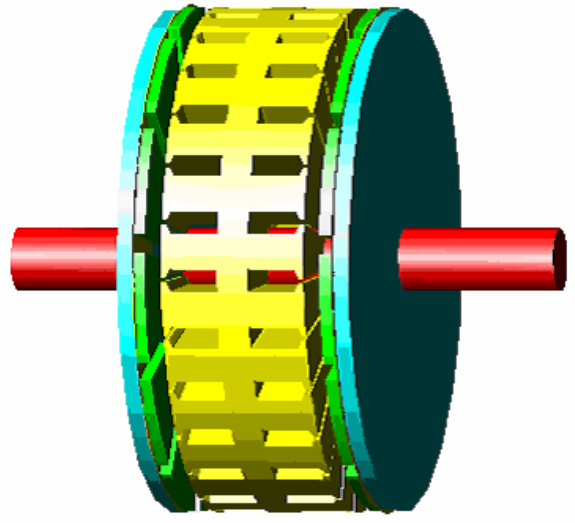

Fig.2 Axial-flux construction with double rotor

passing through the air-gap on the other side of the stator core, going into the pole with opposite polarity on the second disk. The flux returns to the start pole along a similar route to the paths discussed above, as shown in Fig. 3.

The main function of the stator yoke in this structure is for mechanical support and the magnetic length can be minimized. This will reduce the stator MMF requirements and also reduce the stator loss. As the relative permeability of the permanent magnets is close to air, the influence of low permeability SMC is reduced, relative to non permanent magnet designs.

\section{BASIS FOR PM GENERATOR DESIGN}

The task of an electrical machine design is to determine the machine topology, choose necessary materials to be used, and decide the sizes in various sections, based on the requirements of power and torque. These requires magnetic circuit calculations and verification of the performance equation.

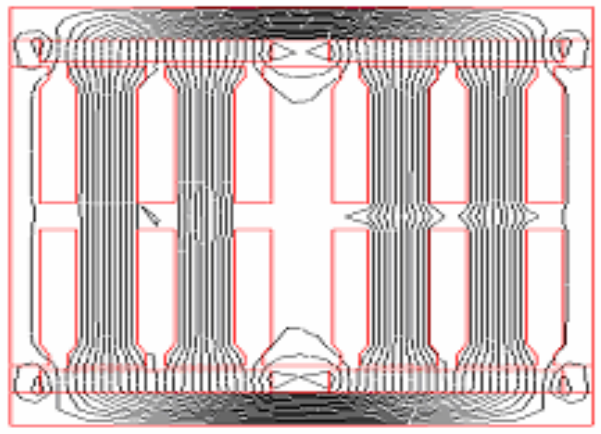

Fig.3 Flux paths of the double rotor axial-flux structure

\section{A. Magnetic Circuit Calculations}

Fig.4 shows the equivalent magnetic circuit derived on the basis of the material properties and the demagnetization curve of permanent magnets. In the equivalent magnetic circuit, $\Phi_{r}$ is the intrinsic flux, which is constant at constant temperature; $\Phi_{0}$ is called the inner leakage flux; $\Phi_{m}$ is the flux produced by magnet pole; $\Lambda_{0}$ is the inner leakage permeance, which depends on the dimensions and property of the magnet; $F_{m}$ is the MMF provided by the magnets to the total magnetic circuits; $\Phi_{\sigma}$ is the leakage flux; $\Lambda_{\sigma}$ is the leakage permeance of the magnetic pole, which is usually difficult to calculate using an analytical method; $\Phi_{\delta}$ is the air-gap flux; $\Lambda_{\delta}=\Phi_{\delta} / \sum F$ is called the main permeance, through which the air-gap flux passes from magnet pole into armature core; $F_{a d}$ is the d-axis armature reaction MMF, $\sum F$ is the total MMF expended in the magnetic circuits.

To solve the equivalent magnetic circuit, a predetermined working point for the magnet is assumed and checked at the end of the calculation. As the calculations always involve non-linear circuits and saturation in electrical machines, iteration for the calculation of working point of the magnet is required.

\section{B. Leakage Permeance CalculationsBy Finite Element Method}

The calculation of leakage permeance, which depends on the leakage paths, is very difficult since the distribution of leakage flux is complex. To accurately

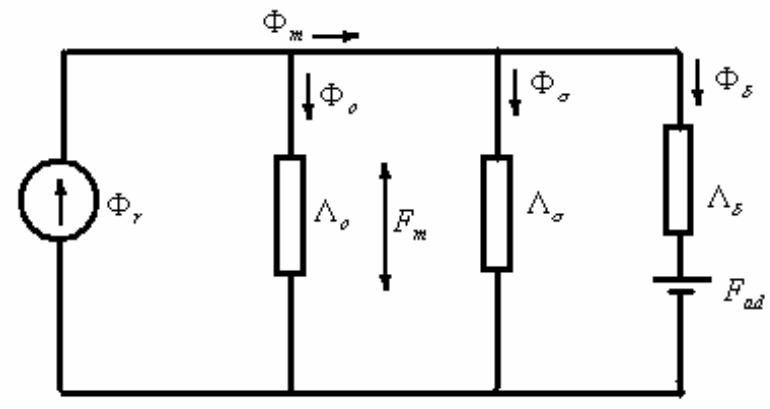

Fig. 4 Equivalent magnetic circuit of PM machines 
calculate the leakage flux, the finite element (FE) method is essential. Because of the structural complexity in axial-flux machines, 3-D analysis is required. Fig. 5 shows the mesh of a 3-D model.

3-D FE calculations are time consuming and difficult to apply in preliminary designs and design optimization studies, where large amounts of calculations exist. To overcome this difficulty, the leakage flux calculation is based on analytical formulas, with a modification coefficient, which is obtained from 3-D and simplified 2D FE computations to increase the accuracy. Thus, the modified leakage permeance is

$$
\Lambda_{\sigma}=k_{\sigma} \Lambda_{\sigma a}
$$

where $k_{\sigma}$ is the leakage modification coefficient of leakage permeance; $\Lambda_{\sigma a}$ is the leakage permeance calculated by analytical formula. Fig. 6 shows the leakage modification coefficient change with different radial magnet lengths, when the air-gap length is fixed at $0.8 \mathrm{~mm}$ and magnetizing lengths of the magnet are $6 \mathrm{~mm}$ and $4 \mathrm{~mm}$.

\section{Design Equitions}

The air-gap flux per pole in a PM wind generator can be expressed as

$$
\Phi_{\delta}=\frac{b_{o} B_{r} A_{m}}{\sigma_{o}}
$$

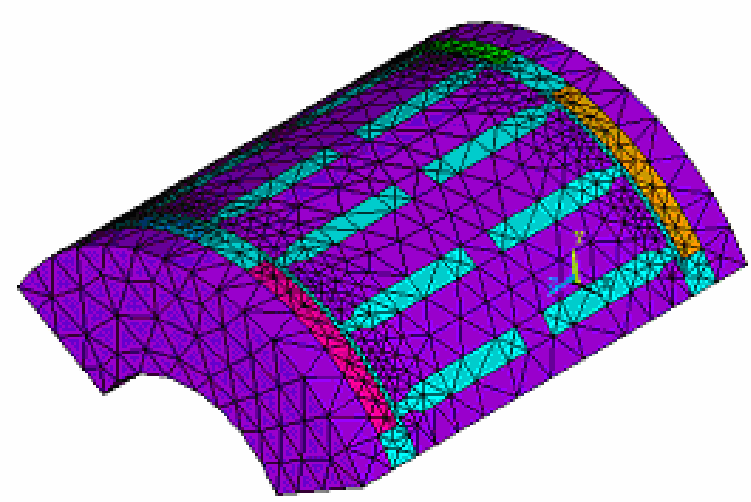

Fig. 5 3-D meshes of an axial-flux PM machine

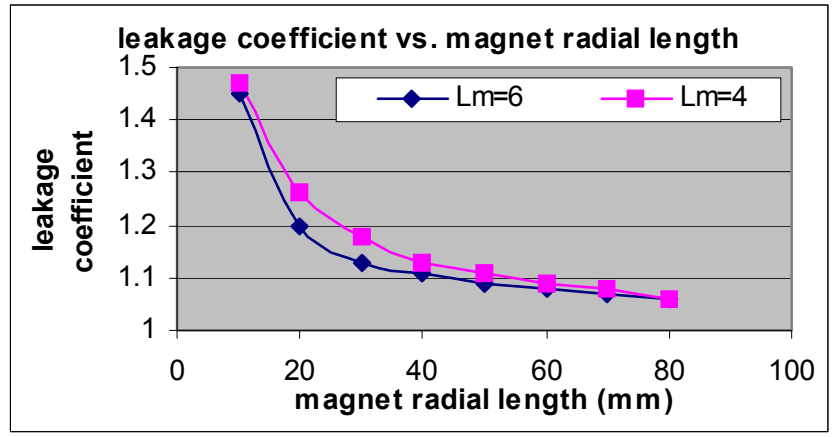

Fig. 6 Leakage flux coefficient where $B_{r}$ is the remanence of the magnet; $A_{m}$ is the magnet cross area; $b_{o}$ is the magnet working point, and $\sigma_{o}$ is the leakage coefficient of the magnet, which can be computed in the magnetic circuit calculation. The fundamental flux per pole is

$$
\Phi_{\delta 1}=k_{f a i} \Phi_{\delta}
$$

where

$$
k_{f a i}=\frac{\Phi_{\delta 1}}{\Phi_{\delta}}=\frac{8}{\pi^{2} \alpha_{i}} \sin \frac{\alpha_{i} \pi}{2}
$$

is the flux waveform coefficient, and $\alpha_{i}$ is the effective polearc coefficient. Then the excitation voltage can be expressed as

$$
E_{f}=4.44 f N_{p h} K_{w} \Phi_{\delta 1}
$$

where $N_{p h}$ is the number of turns per phase, and $K_{w}$ is the fundamental winding coefficient.

To solve the equivalent magnetic circuit, the d-axis armature reaction MMF $F_{a d}$ is required,

$$
F_{a d}=0.9 m \frac{N_{p h} K_{w}}{p} k_{a d} I_{d}
$$

where

$$
k_{a d}=\frac{\alpha_{i} \pi+\sin \alpha_{i} \pi}{4 \sin \frac{\alpha_{i} \pi}{2}}
$$

is the d-axis armature reaction coefficient.

The synchronous reactance $X_{s}$ is related to the armature reaction reactance $X_{m}$ and leakage reactance $X_{l}$ as

$$
X_{s}=X_{m}+X_{l}
$$

where $X_{m}$ can be calculated by

$$
X_{m}=\frac{E_{f} F_{a d}}{I_{N} \sum F} k_{a d}
$$

where $I_{N}$ is the rated current. The leakage reactance can be calculated in terms of slot, tooth tip, harmonic and winding overhang leakage flux paths and by the same formula as in induction motors.

The steady-state terminal voltage of a stand-alone PM wind generator can be determined by considering its per-phase equivalent circuit and phasor diagram. For a machine with 
negligible saliency and lagging power factor, this can be expressed as

$$
\begin{aligned}
V_{a}= & \sqrt{E_{f}^{2}-\left(I_{a} X_{s} \cos \varphi-I_{a} R_{a} \sin \varphi\right)^{2}} \\
& -I_{a} X_{s} \sin \varphi-I_{a} R_{a} \cos \varphi
\end{aligned}
$$

In the case of leading power factor, the terminal voltage is expressed as

$$
\begin{aligned}
V_{a}= & \sqrt{E_{f}^{2}-\left(I_{a} X_{s} \cos \varphi+I_{a} R_{a} \sin \varphi\right)^{2}} \\
& +I_{a} X_{s} \sin \varphi-I_{a} R_{a} \cos \varphi
\end{aligned}
$$

According to the results from the equivalent magnetic circuit calculation, the flux densities in various sections, such as air-gap flux density, stator tooth flux density and stator yoke flux density, can be obtained. Therefore, the performance calculations, such as core losses and efficiency, can be done.

\section{DESIGN CONSIDERATIONS AND COMPARISONS}

Machine design is a synthetic knowledge application of machine theory and manufacturing experience. Many parameters and dimensions will influence the design results. Based on the discussion and analysis in the previous section, the design procedure is programmed, which can be used to design PM wind generators of different topologies. For the convenience of discussing design considerations and comparisons, Table 1 gives the design results of wind generators for four different structures, which are the double rotor axial-flux machine with SMC slotted stator, Torus axialflux machine with spirally wound stator, outer rotor radialflux machine with electrical steel lamination stator, and Bergey XL.1. Bergey is an American company that produces a line of small wind machines. Bergey XL.1 is its product, which has an outer rotor radial-flux structure and is redesigned for comparison here. To prove the advantage of using the SMC in a double rotor slotted structure, the Torus machine is chosen as the comparison, as it has an axial-flux structure. Another outer rotor radial flux machine with different output voltage is designed to verify the program.

12 poles are chosen for all machines to reduce the machine diameter. As the number of slots is limited, the number of slot per pole per phase $q$ is quite small, and has to be fractional with $q=3 / 4$ to reduce harmonics. Increasing slot numbers is mainly limited by the minimum tooth width.

Air-gap flux density in Torus machine is very small, as the total air-gap length in this kind of machine is large. The flux density in the SMC machine is $0.6614 \mathrm{~T}$, being close to the value in the radial flux machines with laminated cores. This is obtained for two reasons. The air-gap length is shortened in the SMC machine, being $0.8 \mathrm{~mm}$. Another reason is that the flux path in the core in this machine is also shorter compared to the radial flux machines. Comparing the slot height in those machines can see this result. The slot

\begin{tabular}{|c|c|c|c|c|}
\hline & SMC & Torus & $\begin{array}{l}\text { Outer } \\
\text { rotor }\end{array}$ & Bergey \\
\hline Output Power $(\mathrm{kW})$ & 1 & 1 & 1 & 1 \\
\hline Output voltage (V) & 220 & 220 & 220 & 20 \\
\hline Speed (rpm) & 490 & 490 & 490 & 490 \\
\hline Poles & 12 & 12 & 12 & 12 \\
\hline Number of slots & 27 & - & 27 & 27 \\
\hline Inner radius & 51 & 57 & 24 & 24 \\
\hline Outer radius & 79.6 & 142.5 & 87.9 & 87.9 \\
\hline Turns per slot & 260 & 70 & 256 & 24 \\
\hline Air-gap flux density (T) & 0.661 & 0.342 & 0.663 & 0.663 \\
\hline Air-gap length (mm) & 0.8 & 6 & 1 & 1 \\
\hline Magnet length (mm) & 4 & 8 & 4 & 4 \\
\hline Total length (mm) & 91.5 & 59.8 & 46 & 46 \\
\hline Stator resistance $(\Omega)$ & 5.28 & 4.54 & 11.04 & 0.094 \\
\hline Armature reactance $(\Omega)$ & 32.52 & 9.634 & 48.84 & 0.429 \\
\hline Torque/volume $\left(\mathrm{Nm} / \mathrm{m}^{\wedge} 3\right)$ & 25943 & 9570 & 25733 & 26189 \\
\hline Torque/weight $(\mathrm{Nm} / \mathrm{kg})$ & 3.399 & 1.237 & 3.256 & 3.313 \\
\hline Magnet weight (kg) & 0.579 & 5.692 & 0.571 & 0.571 \\
\hline Copper weight (kg) & 2.089 & 1.795 & 2.184 & 2.252 \\
\hline Core weight (kg) & 3.495 & 9.377 & 3.717 & 3.717 \\
\hline Total loss (W) & 240.3 & 231.9 & 207.7 & 210.3 \\
\hline Copper loss (W) & 72.74 & 62.52 & 76.05 & 78.43 \\
\hline Iron loss (W) & 91.74 & 93.17 & 55.45 & 55.45 \\
\hline Efficiency (\%) & 80.02 & 81.18 & 82.75 & 82.94 \\
\hline
\end{tabular}
height in SMC machine is $30 \mathrm{~mm}$, and the slot height in radial flux machines is $35 \mathrm{~mm}$.
Table 1 Design results for four structures

For the SMC machine and radial-flux machines, the magnet length is $4 \mathrm{~mm}$, which can make the magnet operating point work above the knee point to prevent irreversible demagnetization. The Torus machine requires a longer magnet length, as its equivalent air-gap length is large.

In this design the SMC machine has a slightly higher iron loss compared to the radial-flux machines because of the inherent drawback of SMC material. Therefore the efficiency of the SMC machine is slightly lower than the efficiency of radial-flux machines although some precautions have been taken. However, the efficiencies are very close.

The best result is that the SMC machine has the highest torque to weight ratio. It is beneficial for the wind energy system, and will reduce the load of the tower.

Table 7.1 provides strong support to our research objective that through careful design, the advantages such as better performance, reduced size and weight, fewer parts and lower cost in axial-flux wind generators using SMC can be obtained. 


\section{DESIGN AND CONSTRUCTION OF THE TEST MACHINE}

The same program as discussed in the previous section is used to design the test machine, which has a double rotor axial-flux structure with SMC armature core. Table 2 shows the design data.

Here the number of poles is equal to 6 , and there are 18 slots on each side of the stator. The inner radius limits the number of poles and slots. The slot width is constant along the radial direction, thus the tooth shape is trapezoidal. The coils are inset into the stator slots to form the three phase double layer windings on each side of the stator, which are then connected in series to form the armature winding. Two cylindrical shapes SMC materials is provided by Hoganas, Sweden. For large production, the stator can be pressed into the desired shape in a mould. To build this test machine, the stator was cut from the solid cylinder SMC at the Clarkson university machine shop, as shown in Fig. 7.

High remanence $\mathrm{NdFeB}$ is ordered for the magnetic poles with remanence being $12(\mathrm{kG})$, coercivity being 11.68 (kOe), and the maximum energy product being 35.2 (MGOe) at room temperature. Each piece of the magnet has the size of $\mathrm{R} 41 \times \mathrm{R} 19 \times 44^{\circ} \times 52^{\circ} \times 4$ in partial annulus shape. The

Table 2 design data for test machine

\begin{tabular}{|c|c|c|c|}
\hline Output power $(\mathrm{kW})$ & 1 & Stator reactance $(\Omega)$ & 2.2 \\
\hline Phase voltage (V) & 24 & Torque/volume $\left(\mathrm{Nm} / \mathrm{m}^{\wedge} 3\right)$ & 11430 \\
\hline Speed (rpm) & 600 & Torque/weight $(\mathrm{Nm} / \mathrm{kg})$ & 1.5043 \\
\hline Number of slots & 18 & Magnet weight $(\mathrm{kg})$ & 0.183 \\
\hline Inner radius (mm) & 20 & Copper weight $(\mathrm{kg})$ & 0.45 \\
\hline Outer radius $(\mathrm{mm})$ & 40 & Core weight $(\mathrm{kg})$ & 0.909 \\
\hline Turns per slot & 110 & Total loss $(\mathrm{W})$ & 100.44 \\
\hline Air-gap flux density (T) & 0.66 & Copper loss $(\mathrm{W})$ & 48.05 \\
\hline Air-gap length $(\mathrm{mm})$ & 0.8 & Iron loss $(\mathrm{W})$ & 11.98 \\
\hline Magnet length $(\mathrm{mm})$ & 4 & Efficiency & 48.88 \\
\hline Stator length $(\mathrm{mm})$ & 50 & Poles & 6 \\
\hline Stator resistance $(\Omega)$ & 8.3 & Rated current (A) & 1.4 \\
\hline
\end{tabular}

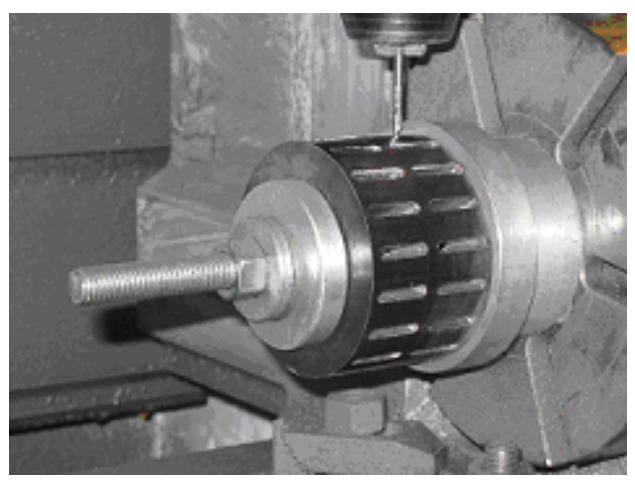

Fig. 7 Stator machining magnets are glued on a solid disk with J-B WELD, which is a strong glue, as shown in Fig. 8.

To further study the influence of the SMC magnetic properties on the flux distribution in the machine, finite element calculations are conducted on the model of the double rotor axial-flux machine with both SMC core and electrical steel core. Fig. 9 gives the result.

As can be seen in Fig. 9, the air-gap flux density of SMC core is very close to the value of electrical steel core, even though the permeability of SMC is lower than that of electrical steel.

The assembly of this kind of machine must be done carefully since large attracting force exists between magnet poles, and between magnet pole and stator core. Fig. 10 shows the assembled stator and rotor. Fig. 11 is the whole machine assembly.

\section{SET UP OF MACHINE EXPERIMENT AND TEST RESULTS}

\section{A. Set up of Machine Experiment}

The designed prototype will operate at variable speed since the wind speed varies. The load of the machine is also changeable according to the requirement of customers. Therefore the machine should be tested with changeable speed and load. To fulfill this requirement, the laboratory set up used for testing the prototype is shown in Fig. 12.

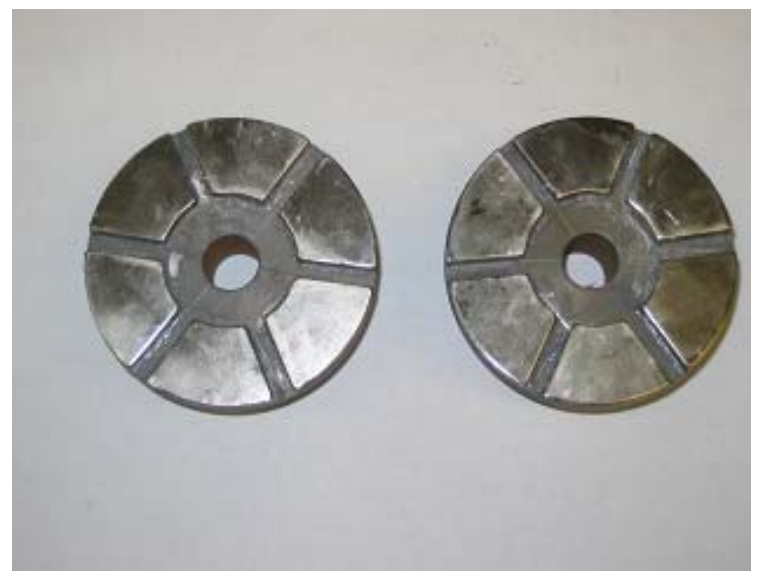

Fig. 8 Magnets glued on the disks

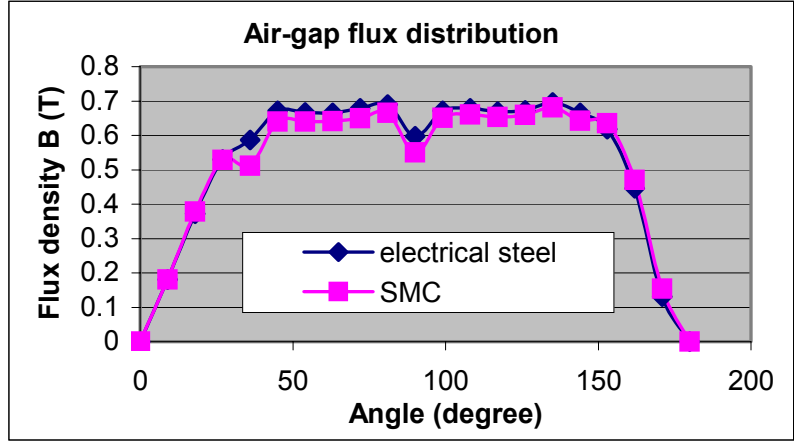

Fig. 9 Flux density with electrical steel and SMC core 


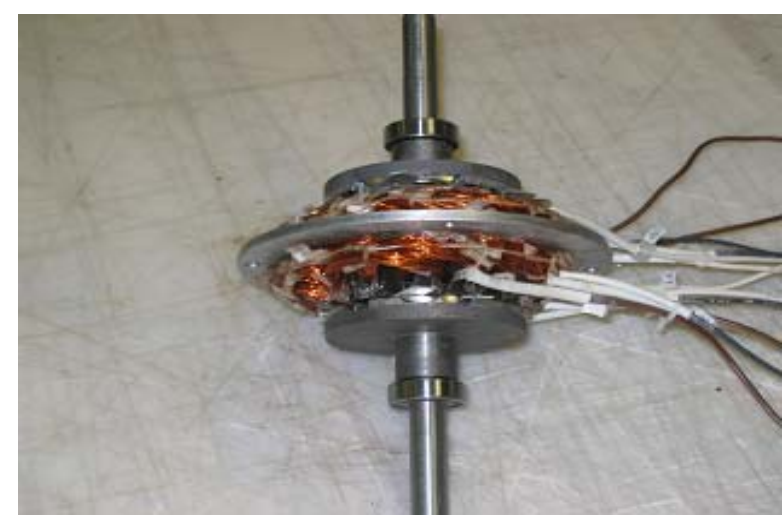

Fig. 10 Assembled stator and rotor

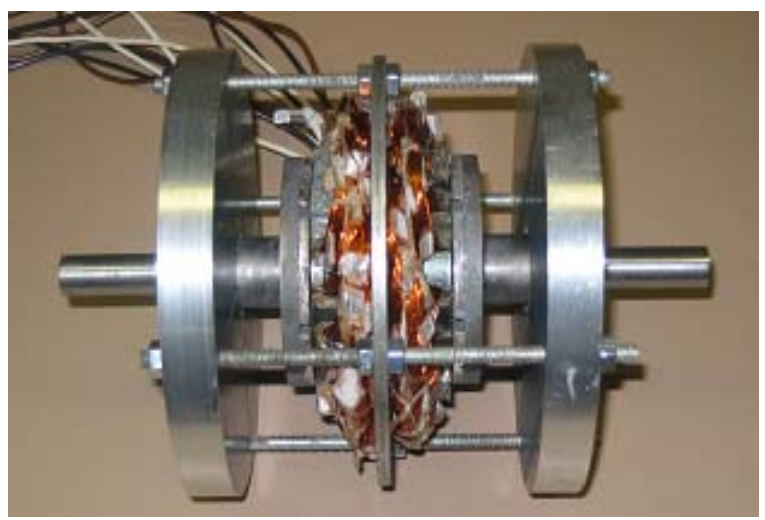

Fig. 11 Assembled machine

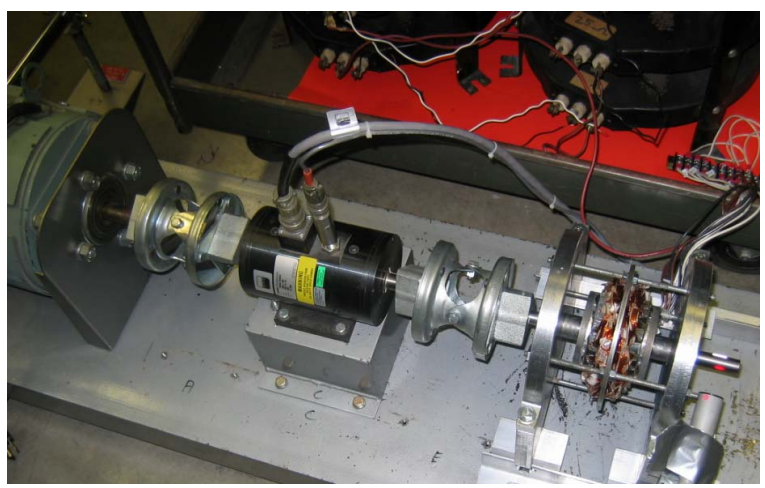

Fig. 12 Experimental set up of the prototype

The tested machine is mechanically connected to a DC motor through a torque transducer. The DC motor is controlled by two adjustable DC voltage sources and operates at adjustable speed to simulate the output of a wind turbine. A three phase adjustable resistor is $\mathrm{Y}$ connected to the terminals of the prototype. The torque is recorded with the torque meter. As the load resistance is adjusted, the loading of the test generator varies. A LeCroy 9304 AM digital oscilloscope is used as the main data acquisition equipment. The voltage and current of the test machine are coupled to the digital oscilloscope through LeCroy voltage probes and Tektronix TM502A current probe amplifier. The rotational speed of the tested machine is recorded through a Hampden tachometer. After the experiment, the recorded data is transferred to the main computer for data processing.

\section{B. No-load Voltage vs. Speed}

Fig. 13 shows that the no-load voltage varies with the rotor speed for both the calculated and test results There is good agreement between the calculated and test value.

The calculated stator phase resistance is $8.3 \Omega$, and the measured value is $8.8 \Omega$. The error comes from the end length calculation of the stator coil. As the radius of the prototype is small, the end length of the coil takes up a large part of the total length of the stator winding, which makes the resistance calculation inaccurate.

\section{No-load Loss vs. Speed}

Fig. 14 shows the characteristic of the no-load loss vs. speed, which includes the mechanical and core losses. The calculated no-load loss is $51.41 \mathrm{~W}$ at $600 \mathrm{rpm}$, and the measured value is $53 \mathrm{~W}$. The calculated core loss is $11.98 \mathrm{~W}$. The separation of core loss from the total loss from measurements is difficult in this machine and needs further work.

\section{Output Power vs. Speed}

Fig. 15 shows the change of output power vs. speed. The rated current is defined at $1.4 \mathrm{~A}$. It can be seen that the generator will capture more energy as the wind speed increases.

\section{E. Output Voltage vs. Current}

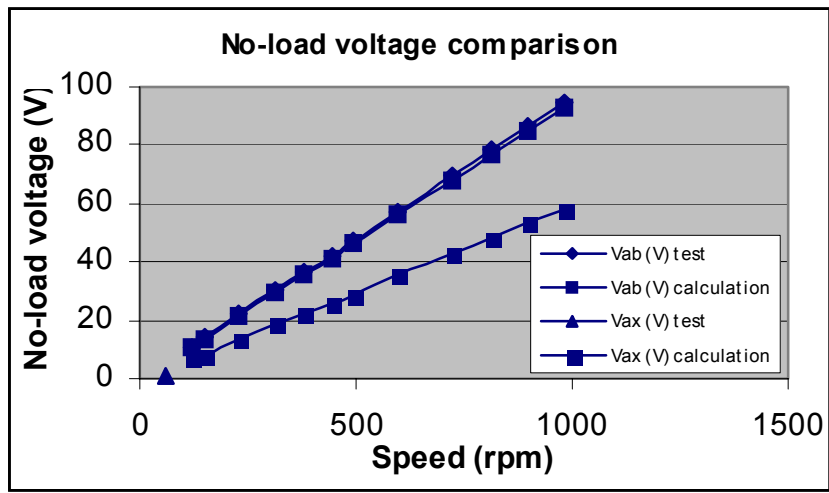

Fig. 13 No-load voltage comparison

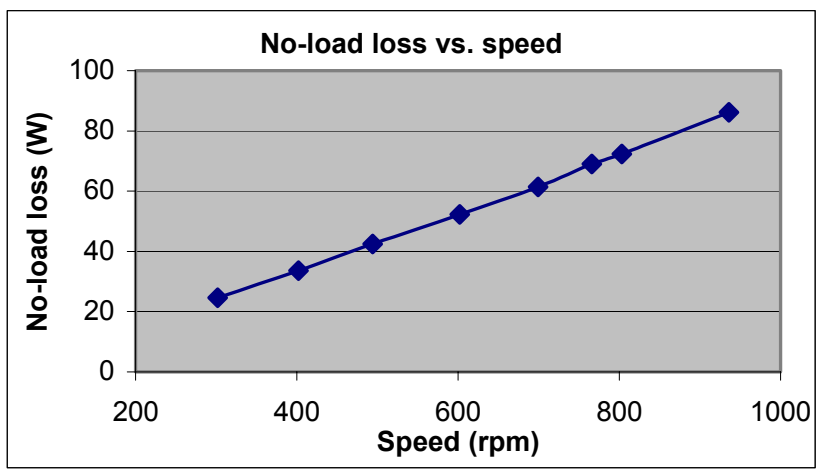

Fig. 14 no-load loss vs. speed 


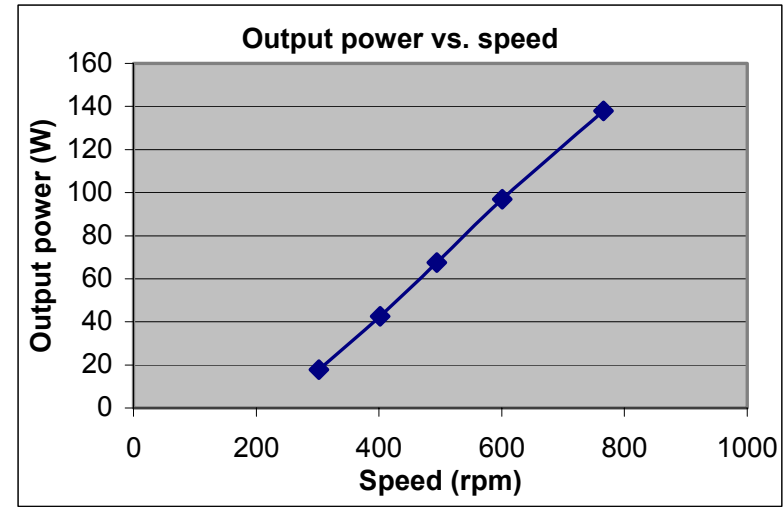

Fig. 15 Output power vs. speed

Fig. 16 shows the output voltage vs. output current at different speeds. As the current or load increases, the voltage drops correspondingly. From the viewpoint of design, the lower the voltage drop, the better the generator performance. This is essentially voltage regulation, which is defined as the ratio of the voltage drop to the rated voltage.

\section{F. Voltage Waveform}

Fig. 17 shows the recorded phase voltage waveform, which is not sinusoidal. High harmonics exists in this machine as the armature coil is difficult to short pitch and the number of slots per pole per phase is unity. However, it is good for directly driven wind generators. A rectifier is required in such wind systems.
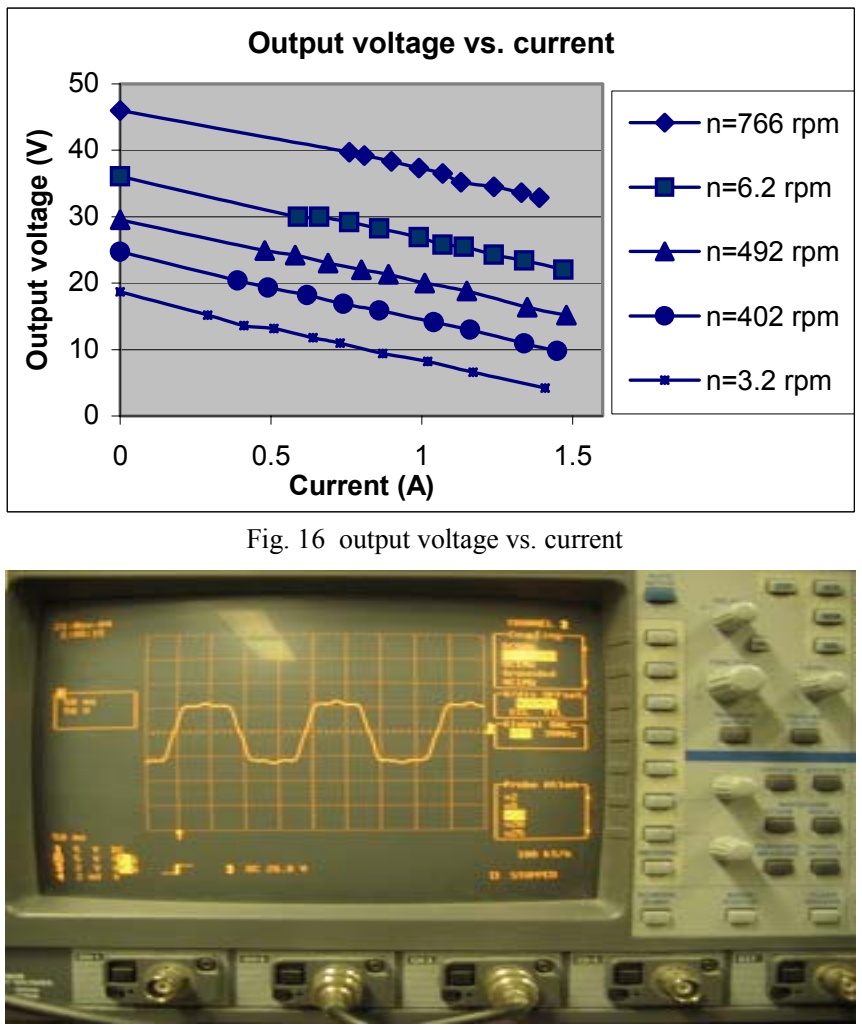

Fig. 17 phase voltage wave form

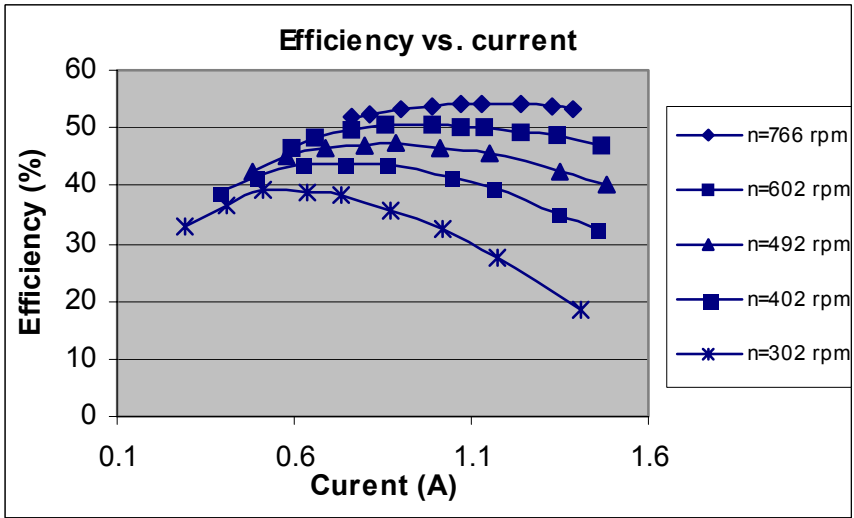

Fig. 18 efficiency vs. current

\section{G. Efficiency vs. Current}

Fig. 18 shows the efficiency vs. current at different speeds. The calculated efficiency is $48.9 \%$ at $600 \mathrm{rpm}$, and the measured value is $48.3 \%$, which is close to each other. The machine efficiency is not high but reasonable as small machines inherently have low efficiency.

\section{CONCLUSION}

Double rotor axial-flux PM generators with slotted cores have many advantages over the machines with other topologies in efficiency, total length, and torque/ volume ratio. The core manufacturing is a crucial issue in this kind of machine.

SMC materials have the potential to be applied to electrical machines, particularly those that are difficult to manufacture with laminations. However, directly replacing electrical steel with SMC is not reasonable.

The combination of axial-flux structure and property of SMC can develop new PM wind generator designs with improved performance, if proper design precautions are taken.

The comparable design of four structure machines and a construction of a prototype have proved the research objective.

\section{REFERENCES}

[1] Yicheng Chen, pragasen Pillay, and Azeem Khan, "PM wind generator comparison of different topologies," Conference Record of the 2004 IEEE Industry Applications Society, pp 800-807, October 2004.

[2] L.O. Hultman and A.G. Jack, "Soft magnetic composotes-materials and applications," Conference Record of the 2003 IEEE Industry Applications Society, pp 516-522, October 2003.

[3] T. Henneron, S. Clenet, Jcros, and P. Viarouge, " Evaluation of 3D finite element method to study and design a soft magnetic composite machine," IEEE Transactions on magnetics, Vol. 40, NO. 2, pp 786789, March 2004.

[4] Y. Guo, J.G. zhu, Peter A. Watterson, and W. Wu, "Comparative study of 3-D flux electrical machines with soft magnetic composite cores," IEEE transactions on Industry Applications, Vol. 39, No. 6, pp 16961703, November/December 2003.

[5] E. Spooner and A.C. Williamson, "Direct coupled, permanent magnet generators for wind turbine applications," IEE Proceedings, Electric Power Applications,. Vol. .143, No. 1, pp. 1-8, January,1996 